这里 $i_{l}$ 满足 $0 \leqslant i_{l} \leqslant k$, 而当 $i_{l}=0$, 认为上式中的 $\lambda_{m_{0}}=\lambda_{l}$ 。 上面 $a_{1}, a_{2}$ 的表达式正是我们 在文献 [8] 中所见到的. 此外,关于这些无穷级数的收玫性问题, 我们尚可作些探讨, 这里不 再赘述.

\title{
考文 战
}

[ 1 ] Dunford, N. \& Schwartz, J. T., Linear Operators, Part III, 1971.

[2] Turmer, R. E. L., J. Math. Anal. Appl., 13 (1966), 447-457.

[ 3 ] Schwartz, J. T., Pacific J. Math., 4 (1954), 415-458.

[. 4 ] Kramer, H. P., Pacific J. Math., 7 (1957), 1405-1435.

[5] Clark, C., Pacific J. Math., 25 (1968), 59-70.

[6] Singer, I., Bases in Banach Space I, 1970.

[7] 王康宁、关型直, 中国科学, 1974, 4, 335-350.

[8] Dirac, P. A. M., The Principles of Quantum Mechanics, Oxford, 1958.

\section{用于密度梯度管的中空玻璃小球标定 的新方法一一流体动力学方法}

\author{
吴大诚霍军军* \\ (成 都工学院)
}

\section{一、引}

密度梯度管技术由于仪器简单、测定迅速、精确度高,因此广泛应用于化学和物理学, 是测 定固体和液体密度的一种重要实验方法 ${ }^{[1]}$ 。近年来此法在高分子科学的应用中得到了发展, 甚至有专门的密度梯度管实验室建立 ${ }^{[2]}$.

密度梯度管的制备, 可采用董履和等 ${ }^{[3]}$ 的方法, 很易得到线性梯度。梯度管的标定有几 种方法, 其中最常采用密度已知的中空玻璃小球作为密度位置的浮标, 从而标定出梯度管的密 度一位置关系. 因此, 中空玻璃小球表观密度绝对值的测定, 是密度梯度管标定的关键问题之

中空玻璃小球密度的测定, 一般采用沉浮法, 即密度滴定法 ${ }^{[4]}$. 此法借滴定改变液体介质 的组成 (浓度), 从而改变其密度, 同时观察小球在介质中的运动. 当小球密度大于介质密度 时, 小球在介质中下沉; 反之, 小球在介质中上升; 当二者密度相等时, 小球将悬浮在介质中不 动. 因此, 根据液体介质的密度, 以及小球运动方向的变化, 可确定出小球的密度. 借改变液 体介质的温度来改变密度, 再作沉浮观察以确定小球密度, 是上述方法的一个变种, 称为温度 交错法 ${ }^{[4]}$. 也有人建议采用刚配制好的标准线性密度梯度管,反过来标定小球密度 ${ }^{[5]}$.

从流体力学的观点看来, 这些方法主要应用了流体静力学的 Archimedes 原理, 至于流体 动力学方面则只应用了关于小球运动方向的定性结果. 在流体动力学中, 关于小球在粘性介 质中的运动, 已有大量的理论和实验研究, 但主要的注意集中在小球运动与介质粘性的关系

*車忠碧、析忠贺同志参加部分实验工作. 
上. 小球在介质中的运动速度, 对于小球与介质密度差的关系, 有人曾由 Stokes 方程预示应 呈正比 ${ }^{[6]}$. 然而, 考虑到介质密度改变必然伴随有粘度变化, 还有阻力系数不等于 1 的非理想 情况的偏差,这种简单关系显然不能反映实际情况. 为此, 本工作根据改正的 Stokes 方程, 拟 定了一种测定小球与介质密度差的流体动力学方法, 并将所得的小球表观密度与上述已知的 三种标定方法进行了比较.

\section{二、测定原理}

按照改正的 Stokes 方程 ${ }^{[7]}$, 在粘度 $\eta$ 和密度 $\rho_{L}$ 的液体介质中, 半径 $r$ 和密度 $\rho_{s}$ 的固体小 球于重力作用下达到定常运动状态时,其速度将为:

$$
u=2 / 9 \frac{r^{2}\left(\rho_{s}-\rho_{L}\right) g}{\eta K_{D}},
$$

式中 $g$ 是重力加速度, $K_{n}$ 是陈景尧 ${ }^{[8]}$ 建议的阻力系数, 是雷诺数 $R e=2 r \rho_{l, v} / \eta$ 的函数.

当液体介质不是无限广延, 而盛于内径为 $2 R$ 、高度为 $H$ 的圆柱形长筒中,阻力系数 $K_{D}$ 般说来应是 $R e 、 r / R$ 和 $r / H$ 的某种函数. 这方面已有大量的研究，但理论上尚未完全解决. 然而在适当的条件下，管壁和端面效应可完全不予考虑，下面讨论中将对此问题作适当的说 明. 因此仍可简单地采用式 (1) 的关系,并重新改写为:

$$
K_{D} \eta v=A \rho_{S}-A \rho_{L},
$$

式中 $A=2 / 9 r^{2} g$, 是决定于小球半径的常数。由此可知,在同一圆筒中对于同一玻璃小球,改 变筒内液体介质的密度 $\rho_{L}$ 和粘度 $\eta$, 经实验求出小球在介质中的定常速度 $v$, 再根 据下文中 介绍的方法计算出 $K_{D}$ 值; 然后, 用 $K_{D} \eta v$ 对 $\rho_{L}$ 作图,所得应为一条斜率为 $A$ 的直线，此直线与 $\rho_{L}$ 轴 $\left(K_{D} \eta \nu=0\right)$ 的交点, 即是所求的固体小球密度之值 $\rho_{s}$.

\section{三、实验与计萛方法}

1. 小球运动速度的实验测定

本工作实验置与普通落球法测定液体粘度所用相同. 盛液体介质的圆形长筒 $R=9$ 厘 *, $H=30$ 厘米. 因同一小球在介质体系中需多次下沉或上升重复测定, 为了减少取出或放 人小球而引起介质扰动, 除每次测定前应将液体静置15分钟外,还在圆筒上下端附加漏斗形导 管,下端漏斗与一玻管相通，以便在下端放入或取出小球。测定时温度控制在 $20 \pm 0.1^{\circ} \mathrm{C}$.

用两台测高仪对准圆筒，在筒上标出 20 厘米左右的距离，用测高仪准确测定至 0.01 厘 米. 然后, 用停表通过测高仪目镜测定小球通过这段距离的时间, 准确至 0.1 秒. 由此可得出 下沉或上升速度,并规定下沉时速度为正上升为负．对介质的每一种浓度,使用同一小球测定 10 次, 取其算术平均值为 $v$ 。

2. 液体介质性质的测定和计算

本工作采用氯化钻、乙二醇和丙三醇的水溶液，作为液体介质。水溶液在 $20^{\circ} \mathrm{C}$ 下对钠黄 光的折光指数 $n$ 由阿贝折光仪测定. 溶液的密度 $\rho_{L}$ 和粘度 $\eta\left(20^{\circ} \mathrm{C}\right)$ 的数值, 可由对应的 $n$ 值 用内插法求出。 $n-\rho_{I}-\eta$ 的标准数据参见文献 $[9]$.

3. 小球运动的雷诺数及阻力系数的测定和计算

小球直径用读数显微镜测定, 可精确至 $1 \times 10^{-3}$ 厘米.

雷诺数 $R e=2 r \rho_{L} v / \eta$, 根据上述测定值和计算值由定义算出。 
阻力系数 $K_{D}$ 作为 $R e$ 的函数，在本文中建议采用文献 $[10,11]$ 中经整理的实验值，用作 图法求得.

\section{四、实验结果和讨论}

1. 对于某一小球进行的实验测定和计算结果汇总于表 1 .

表 1 某一小球的实验数据

(小球直径 0.358 厘米,乙二醇水溶液)

\begin{tabular}{|c|c|c|c|c|c|c|}
\hline 实验编号 & $n$ & $\begin{array}{c}\rho_{L} \\
\left.\text { (克/厘米 }{ }^{3}\right)\end{array}$ & $\begin{array}{c}\eta \\
\text { (厘泊) }\end{array}$ & (厘米/秒) & $R e$ & $K_{D}$ \\
\hline 1 & 1.3669 & 1.0438 & 2.44 & 1.70 & 26.1 & 2.40 \\
\hline 2 & 1.3693 & 1.0468 & 2.59 & 1.39 & 20.1 & 2.19 \\
\hline 3 & 1.3709 & 1.0488 & 2.68 & 1.16 & 16.3 & 2.04 \\
\hline 4 & 1.3727 & 1.0511 & 2.81 & 0.960 & 12.9 & 1.92 \\
\hline 5 & 1.3740 & 1.0528 & 2.91 & 0.728 & 9.42 & 1.74 \\
\hline 6 & 1.3755 & 1.0548 & 3.04 & 0.512 & 6.36 & 1.55 \\
\hline 7 & 1.3762 & 1.0558 & 3.10 & 0.374 & 4.56 & 1.44 \\
\hline 8 & 1.3770 & 1.0568 & 3.17 & 0.252 & 3.00 & 1.32 \\
\hline 9 & 1.3778 & 1.0578 & 3.22 & 0.130 & 1.53 & 1.18 \\
\hline 10 & 1.3795 & 1.0600 & 3.38 & -0.246 & 2.76 & 1.29 \\
\hline 11 & 1.3802 & 1.0608 & 3.44 & -0.369 & 4.07 & 1.38 \\
\hline 12 & 1.3810 & 1.0618 & 3.53 & -0.464 & 4.99 & 1.47 \\
\hline 13 & 1.3820 & 1.0631 & 3.58 & -0.556 & 5.92 & 1.55 \\
\hline 14 & 1.3834 & 1.0648 & 3.67 & -0.714 & 7.42 & 1.64 \\
\hline 15 & 1.3850 & 1.0668 & 3.86 & -0.841 & 8.32 & 1.68 \\
\hline 16 & 1.3866 & 1.0688 & 4.15 & -0.982 & 9.03 & 1.72 \\
\hline 17 & 1.3882 & 1.0707 & 4.35 & -1.08 & 9.52 & 1.76 \\
\hline 18 & 1.3906 & 1.0739 & 4.67 & -1.25 & 10.3 & 1.78 \\
\hline
\end{tabular}

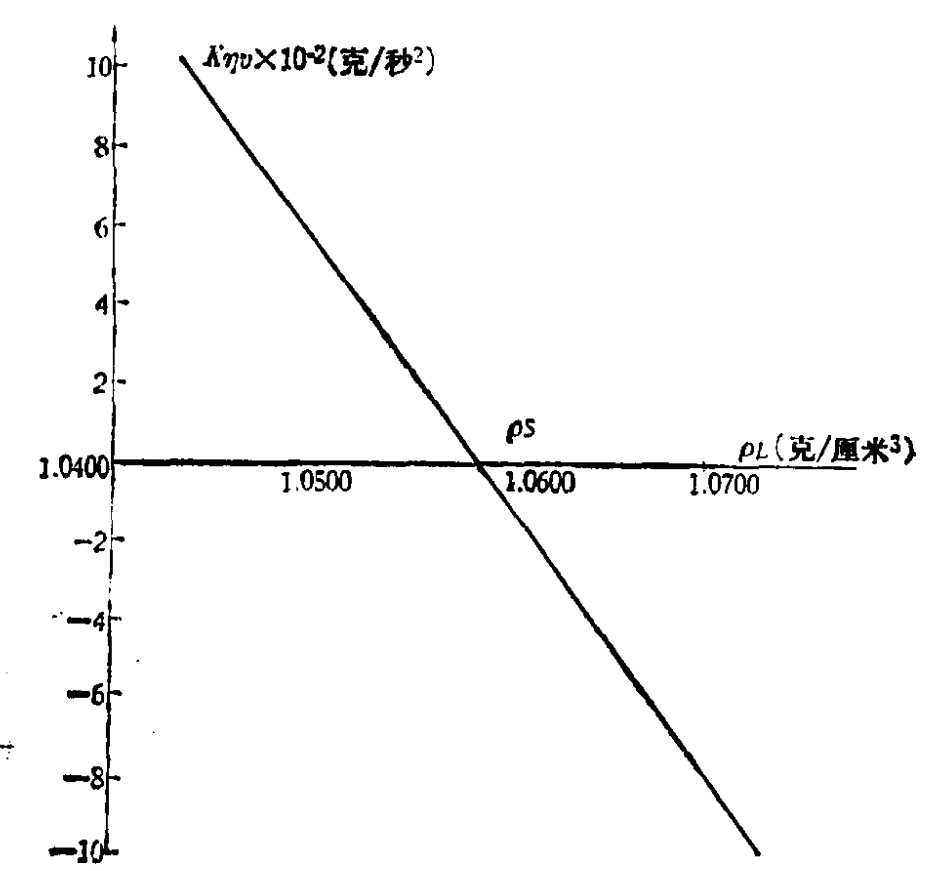

图 1 某一小球的 $K_{D} \eta v-\rho_{L}$ 关系 （由表 1 实验数据得出）

根据表 1 数据, 用 $K_{D} \eta \nu$ 对 $\rho_{L}$ 作图, 呈明显的线性 (图 1), 由此直线与直线 $K_{D} \eta v=0$ 的交点可得出 $\rho_{S}=1.0585$ 克/厘米 ${ }^{3}$.

若用 $v$ 对 $\rho_{L}$ 作图, 并不能得到由文 献 [6]所预示的直线,因而不能简单地求 出 $\rho_{s .}$ 实际上,此种方法只是本文式 (2) 的一种特 例, 在式 (2) 中令 $K_{D}=1$ 和 $\eta=$ 常数, 则可得出 $v-\rho_{I}$ 的线性关系. 但这种假定显然与上述实验值不符。

本工作推荐的流体动力学方法与传 统的小球密度标定方法，对于同一小 球在上述三种体系中的测定结果列于表 2 .

2. 本文中 $K_{D}$ 值在 $0<R e<10$ 范围 内用文献 $[10]$ 实验值, 在 $10 \leqslant R e<100$ 
表 2 小球密度的几种标定方法的结果比较

\begin{tabular}{|c|c|c|c|c|c|c|c|c|c|c|}
\hline \multirow{3}{*}{ 实 䠯 方 法 } & \multirow{3}{*}{ 参考文献 } & \multicolumn{9}{|c|}{ 小球密度实验值 (克/厘米 $\left.{ }^{3}\right)$} \\
\hline & & \multicolumn{3}{|c|}{ 求化钻水湻液 } & \multicolumn{3}{|c|}{ 乙二醉水溶液 } & \multicolumn{3}{|c|}{ 丙三醏水溶液 } \\
\hline & & 1 & 2 & 3 & 1 & 2 & 3 & 1 & 2 & 3 \\
\hline 密度济定法 & [4] & 1.0543 & 1.0591 & 1.0605 & 1.0543 & 1.0588 & 1.0601 & 1.0541 & 1.0589 & 1.0589 \\
\hline 温度交错法 & [4] & 1.0531 & 1.0589 & 1.0601 & 1.0535 & 1.0585 & 1.0601 & 1.0540 & 1.0595 & 1.0608 \\
\hline 制每标准梯度管法 & [5] & 1.0543 & 1.0595 & 1.0607 & 1.0536 & 1.0590 & 1.0602 & 1.0536 & 1.0590 & 1.0603 \\
\hline 流体动力学方法 & [本文] & 1.0536 & 1.0586 & 1.0601 & 1.0535 & 1.0585 & 1.0601 & 1.0535 & 1.0588 & 1.0604 \\
\hline
\end{tabular}

注: $1 、 2 、 3$ 号小球直径分别为 $0.652,0.358$ 和 0.468 厘米.

范围内用文献 [11]实验值. 其原因是在 $R e>12$ 时, 目前尚无可靠的理论值. 由于还存在其它 一些作者的不同实验值,这样的取用难免有一定的任意性。但是,取用目前较可靠的理论值和 某些作者的实验值， $\rho_{S}$ 的测定结果相差极微，只是影响 $A$ 值而已，也就是说，对 $K_{D} \eta v-\rho_{L}$ 作 图所得直线,因选用不同作者的 $K_{D}$, 只影响其直线斜率, 而其 $\rho_{L}$ 轴的截距变化很小. 例如, 在 $0<R e<10$ 范围内, 取用陈景尧 ${ }^{[7]}$ 的理论值或 Maxworthy ${ }^{[12]}$ 与 Pruppacher 等 ${ }^{[10]}$ 的实验值相 比; 在 $10<R e<100$ 范围内，选用 Perry ${ }^{[13]}$ 建议的实验值或 Davies ${ }^{[14]}$ 建议的实验值与 Goldstein $^{[11]}$ 的实验值相比,所得的 $\rho_{s}$ 误差不大于 $1 \sim 2 \times 10^{-4}$ 克/厘米 ${ }^{3}$.

对于适当的小球-液体介质体系，总可以使实验的 $R e<12$, 此时用陈固尧的理论 $K_{D}$ 值， 由此避兔选用实验值引起的疑问。

3. 对于三个直径不同的小球，在三种液体介质中实验测得的 $K_{D} \eta v-\rho_{\mathrm{L}}$ 直线斜率 $A$ ，与其 计篗值相互比较示于表 3 .

表 $3 A$ 的实验值和计算值的比较

\begin{tabular}{|c|c|c|c|}
\hline \multirow{2}{*}{$\begin{array}{c}\text { 小球直径 } \\
\text { (回米) }\end{array}$} & \multicolumn{2}{|c|}{$A$ 的实验值 ${ }^{*}\left(\right.$ 厘米 ${ }^{3} \cdot$ 秒 $^{-2}$ ) } & \multirow{2}{*}{$\begin{array}{c}A \text { 的计算值 } \\
\left(\text { 瘇米 }{ }^{3} \cdot \text { 秒 }^{-2}\right)\end{array}$} \\
\hline & $K_{D}$ 按 $[10,13]$ 计算所得 $A$ 值 & $K_{D}$ 按 $[11]$ 计算所得 $A$ 值 & \\
\hline 0.358 & 6.57 & 6.87 & 6.98 \\
\hline 0.468 & 10.6 & 11.6 & 11.9 \\
\hline 0.652 & 20.7 & 21.3 & 23.1 \\
\hline
\end{tabular}

* 在三种介质体系中的平均值.

事实上,式 (2) 所示的线性关系，不仅仅在完全忽略管壁和端面效应的情况下才成立，而 只缼对于阻力系数 $K_{D}$ ，雷诺数校正与管壁和端面校正没有交叉作用存在，此线性关系即能成 立. 即只要能将 $K_{D}$ 写为以下两项的乘积:

$$
K_{D}=K^{\prime}(R e) \cdot K^{\prime \prime}\left(\frac{r}{R}, \frac{r}{H}\right),
$$

其中 $K^{\prime}(R e)$ 只是 $R e$ 的函数, 即无限广延介质体系中阻力系数的 $R e$ 关系; $K^{\prime \prime}\left(\frac{r}{R}, \frac{r}{H}\right)$ 只 是 小球半径和圆管尺寸的函数，对于同一小球在同一圆管中测定，则一定服从式 (2) 的线性关 系, 只需将直线斜率 $A$ 重新改写为

$$
A=2 r^{2} g / 9 K^{\prime \prime}\left(\frac{r}{R}, \frac{r}{H}\right) .
$$


关于 $K^{\prime \prime}\left(\frac{r}{R}, \frac{r}{H}\right)$ 的具体形式,目前了解不多. 仅就管壁校正 $r / R$ 项而言, 由广泛的实验 研究结果可知 ${ }^{[151}$,一般说来,管壁校正除决定于 $r / R$ 外, 还与 $R e$ 有关, 且随 $R e$ 增大而减小,关 系相当复杂. 然而十分幸运的是, 当 $R e$ 大于某一适当数值 $R e_{C}$ 后,管壁校正将不再与 $R e$ 有 关,且校正值会小于 $1 \%$. 若 $r / R=0.05$, 则 $R e_{c}=5 ; r / R=0.10$, 则 $R e_{c}=30$. 因此, 采用 较小的小球时,可完全忽略管壁校正, 但采用较大的小球时, 必须在较高 $R e$ 下测定,才能完全 忽略管壁校正. 至于端面校正, 其理论和实验研究更不充分 ${ }^{[16]}$, 但估计不会超过管壁校正, 因 此与绝大多数实验工作一样, 完全不计此种校正. 由上述讨论可知, 表 2 所示 $A$ 的实验值与理 论值的偏离,除去普通的各项实验误差之外,尚有式 (4) 代表的管壁和端面校正末考虑所引起 的误差.

4. 由式(2) 变换之后可得:

$$
\rho_{S}=K_{D} \eta v / A+\rho_{L},
$$

因此,若采用理论计算的 $A$ 值 $\left(A=\frac{2}{9} r^{2} g\right)$, 则从每一实验对应的数据可根据上式计算出小球 密度 $\rho_{s}$, 由此可实现流体动力学测定密度方法的简化.

对于三种不同体系的三个小球, 由式 (5) 计算所得密度的平均值, 与由式 (2) 作直线外推 的密度值相比,其差一般小于 $2 \times 10^{-4}$ 克/厘米 ${ }^{3}$.

由 9 组 97 个按式 (5) 计算出的密度值与式 (2) 外推值比较, 计算出平均误差为 $\pm 3.5 \times$

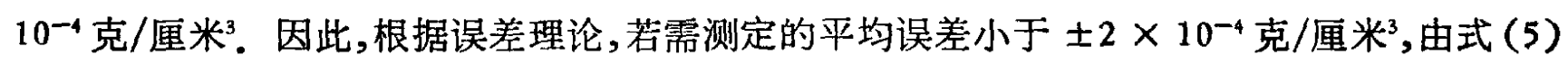
所示的简化方法只需进行 3 次不同浓度介质的测定即可.

5. 测定给定距离的运动时间 $t$, 可计算出小球运动速度 $v$. 对于每一个实验点需测定运动 时间 10 次, 由此可得出平均运动时间 $t$, 从而可计算平均运动速度 $v$, 还可求出运动时间和运 动速度的均方根偏差 $\Delta \bar{i}$ 和 $\Delta \bar{v}$ 。

可以证明,偏差 $\Delta \bar{v}$ 由下式近似得出:

$$
\Delta \bar{v} \approx \frac{\Delta \bar{t}}{t} \cdot v
$$

在较低雷诺数下由于 $\Delta \bar{i}$ 的迅速上升, 而在较高雷诺数下由于 $v$ 的持续增加, 均使得偏差 $\Delta \bar{v}$ 有所增大. 在本工作中, 若令典型条件为: $A=10$ 厘米 ${ }^{3}$ 秒 ${ }^{-2} 、 K_{D}=2.5$ 以及 $\eta=2 \times 10^{-2}$ 泊, 假定要使 $\rho_{S}-\rho_{L}$ 的测定偏差小于 $2 \times 10^{-4}$ 克/厘米 ${ }^{3}$, 则偏差 $\Delta \bar{v}$ 不应高于 $4 \times 10^{-2}$ 厘米/ 秒. 由实验可知,这要求测定时 $R e$ 不应高于 50 .

6. 在上述测定原理中, 曾假定运动是定常的. 根据小球在粘性介质中的运动方程:

$$
\frac{4}{3} \pi r^{3} \rho_{S} \frac{d v}{d t}=\frac{4}{3} \pi r^{3}\left(\rho_{S}-\rho_{L}\right) g-6 \pi r \eta v K_{n},
$$

只有当 $t=\infty$ 时,才能达到定态:

$$
\frac{d v}{d t}=0
$$

由此,推出方程 (1). 若重新将小球运动定常速度写为 $v_{\infty}=2 / 9 \frac{r^{2}\left(\rho_{S}-\rho_{L}\right) g}{\eta K_{D}}$, 当初始条件 $t=$ 0 时 $v=0$, 经时间 $t$ 之后, 小球运动的瞬时速度则将为:

$$
v=v_{\infty}\left[1-\exp \left(-\frac{9}{2} \frac{K_{D} \eta}{r^{2} \rho_{S}}\right) t\right] \text {. }
$$


令 $r \approx 0.2$ 厘米、 $\rho_{S} \approx 1$ 克/厘米 ${ }^{3}, K_{D} \approx 1$ 和 $\eta \approx 2 \times 10^{-2}$ 泊, 则由上式可估算出 $v / v$ 达到 $0.9 、 0.99 、 0.999$ 和 0.9999 的时间 $t$ 分别为 $1.2 、 2.3 、 3.5$ 和 4.6 秒.

在本工作的实验装置中, 小球从挡板 (此处 $v=0$ ) 到计时刻线的距离约 7 厘米,当要求测 定速度与定常速度的偏差小于 0.001 时, 此段距离的运动时间至少应为 3.5 秒左右. 在本工作 的实验测定中,这一条件基本上是满足的.

7. 为实验简便起见, 本工作采用液体的折光指数 $n$ 作为其它性质的折算基础。实验中使 用阿贝折光仪测定, 折光指数精度最好也只能达到 $\Delta n= \pm 1 \times 10^{-4}$, 因此应选择 $\Delta \rho_{L} / \Delta n$ 较 小的体系, 以保证密度 $\rho_{L}$ 的计算精度. 为此, 本工作选用了上述的三种水溶液体系, 当采用差 示折光仪或干涉计测定 $n$ 时, $\Delta n$ 的测定可准确至 $1 \times 10^{-5}$, 使用上述体系计算精度更高, 并 可选用其它适当的体系.

\section{五、结论}

1. 本工作拟定了用于密度梯度管的玻璃小球密度测定的一种新方法一一流体动力学方 法. 用小球速度 $v$ 、液体粘度 $\eta$ 和阻力系数 $K_{D}$ 之乘积 $K_{D} \eta v$ 为参数, 对液体密度 $\rho_{L}$ 作图, 可 得出较好的线性, 由此直线与直线 $K_{D} \eta v=0$ 的交点, 可求出小球密度 $\rho_{s .}$. 对于 $r / R \leqslant 0.05$ 的小球, 测定最好在 $5<R e<50$ 的条件下进行.

2. 使用简化的流体动力学方法测定密度, 只需在 3 种不同浓度介质中进行测定, 再由式 $\rho_{S}=K_{D} \eta v / A+\rho_{\mathrm{L}}$ 计算出其平均值即可.

[1] Oster, G. \& Yamamoto, M., Chem. Rev., 63 (1963), 257.

[ 2 ] Wiley, R., Plastics Technology, 8 (1962), 3, 31.

[3]. Tung, L. H. \& Taylor, W., J. Polym. Sci., 21 (1956), 144.

[4] A. S. T. M., D 1505-68.

[5] Fortuin, J., J. Polym. Sci., 44 (1960), 505.

[6] Dinsdale, A. \& Moore, F., Viscosity and Its Measurement, Chapman and Hall, London, 1962.

[7] 陈䋈光, 中国科学, $1975,4,376$.

[8] 陈量光, 南京工学院学报, $1963,2,77$.

[9] Weast, R., Handbook of Chemistry and Physics, C R S, Ohio, 1974.

[10] Pruppacher, H. \& Steinberger, E., J. Appl. Phys., 39 (1968), 4129.

[11] Goldstein, 8., Modern Developments in Fluid Dynamics, Clarendon, Oxford, 1938.

[12] Maxworthy, T., J. Fluid Mech., 23 (1965), 369.

[13] Perry, R. \& Chilton, C., Chemical Engineers' Handbook, MeGraw-Hill, New York, 1973.

[14] Davies, C. N., Proc. Phys. Soc., 57 (1945), 259.

[15] Fidleris, V. \& Whitmore, R. L., Brit. J. Appl. Phys, 12 (1961), 490.

[16] Maude, A. D., ibid., 12 (1961), 293.

[上接 304 页]

[1] 胡寿永, 古将椎动物与古人类, 8 (1964), 1, 42-63.

[2] 雀枝明, 古将椎动物与古人类, 15 (1977), 1, 59-65.

[ 3 ] 杨镇健, 中国地质学会志, 22 (1942), 3-4, 293-309.

[ 4 ] Gilmore, C. W., Bull. D. S. Nat. Mus., 110(1920), 1-159.

[5] Steel, R., Handbuch der Paläoherpetologie, 1970, Teil. 14, 1-87.

[6] Janenseh, W., Palaeontographica Suppl-Bd. 7, 1925, 1-275.

[ 7 ] 董枝明等, 古零椎动物与古人类, 15 (1977), 4, 307-312. 